\title{
Two Seizure-Onset Types Reveal Specific Patterns of High-Frequency Oscillations in a Model of Temporal Lobe Epilepsy
}

\author{
Maxime Lévesque, Pariya Salami, Jean Gotman, and Massimo Avoli \\ Montreal Neurological Institute and Department of Neurology and Neurosurgery, McGill University, Montréal, Quebec, Canada H3A 2B4
}

High-frequency oscillations (HFOs; $80-500 \mathrm{~Hz}$ ) are thought to mirror the pathophysiological changes occurring in epileptic brains. However, the distribution of HFOs during seizures remains undefined. Here, we recorded from the hippocampal CA3 subfield, subiculum, entorhinal cortex, and dentate gyrus to quantify the occurrence of ripples $(80-200 \mathrm{~Hz})$ and fast ripples $(250-500 \mathrm{~Hz})$ during low-voltage fast-onset (LVF) and hypersynchronous-onset (HYP) seizures in the rat pilocarpine model of temporal lobe epilepsy. We discovered in LVF seizures that (1) progression from preictal to ictal activity was characterized in seizure-onset zones by an increase of ripple rates that were higher when compared with fast ripple rates and (2) ripple rates during the ictal period were higher compared with fast ripple rates in seizure-onset zones and later in regions of secondary spread. In contrast, we found in HYP seizures that (1) fast ripple rates increased during the preictal period and were higher compared with ripple rates in both seizure-onset zones and in regions of secondary spread and (2) they were still higher compared with ripple rates in both seizure-onset zones and regions of secondary spread during the ictal period. Our findings demonstrate that ripples and fast ripples show distinct time- and region-specific patterns during LVF and HYP seizures, thus suggesting that they play specific roles in ictogenesis.

\section{Introduction}

High-frequency oscillations (HFOs; $80-500 \mathrm{~Hz}$ ) are recorded in the EEG of temporal lobe epilepsy patients and in animal models mimicking this condition (Engel and da Silva, 2012; Jefferys et al., 2012). HFOs occur in limbic structures such as the hippocampus and entorhinal cortex, as well as in the neocortex, and they are thought to reflect the activity of dysfunctional neural networks that underlie and sustain epileptogenesis (Bragin et al., 2004; Jacobs et al., 2009, 2010; Ibarz et al., 2010; Jiruska et al., 2010a; Wu et al., 2010; Lévesque et al., 2011). HFOs are also better markers than interictal spikes to identify the seizure-onset zone, independently of the underlying pathology (Jacobs et al., 2008, 2009; Crépon et al., 2010).

HFOs recorded from the hippocampal CA1 and CA3 subfields augment during the transition from preictal to ictal state in the in vitro low- $\mathrm{Mg}^{2+}$ model of epileptiform synchronization (Khosravani et al., 2005). Bragin et al. (2005) also found that HFOs increase in amplitude, duration, and frequency in the dentate gyrus at the onset of seizures occurring spontaneously in vivo in epileptic rats that have received a unilateral hippocampal injection of kainic acid. In keeping with these experimental data, Zijlmans et

\footnotetext{
Received 0ct. 7, 2011; revised June 6, 2012; accepted July 4, 2012.

Author contributions: M.L.,P.S., J.G., and M.A. designed research; M.L. and P.S. performed research; M.L. and P.S. analyzed data; M.L., P.S., J.G., and M.A. wrote the paper.

This work was supported by the Canadian Institutes of Health Research (Grants 8109, 74609, and 102710). M.L. was the recipient of a postdoctoral fellowship from the Savoy Foundation.

The authors declare no competing financial interests.

Correspondence should be addressed to Dr. Massimo Avoli, Montreal Neurological Institute, 3801 University Street, Montréal, QC, Canada, H3A 2B4. E-mail: massimo.avoli@mcgill.ca.

DOI:10.1523/JNEUROSCI.5086-11.2012

Copyright $\odot 2012$ the authors $\quad 0270-6474 / 12 / 3213264-09 \$ 15.00 / 0$
}

al. (2011) recently observed an increase in the percentage of time occupied by HFOs during the transition from preictal to ictal activity in epileptic patients, using time windows of $10 \mathrm{~s}$ before and $5 \mathrm{~s}$ after seizure onset; however, no significant increase in HFOs could be identified 1, 5, and 15 min before seizure onset in another clinical study (Jacobs et al., 2009).

The temporal distribution of HFOs shortly before and during seizures is therefore undefined. In addition, we ignore whether ictal HFOs are differently expressed in seizure-onset zones compared with other areas of the brain. Finally, the temporal and spatial distribution patterns of HFOs in different seizure-onset patterns remain unclear. Here, we addressed HFO dynamics across space and time during spontaneous seizures using chronic multichannel local field potential (LFP) recordings in the rat pilocarpine model of temporal lobe epilepsy. Specifically, we analyzed the occurrence of two different HFO categories [i.e., ripples $(80-200 \mathrm{~Hz})$ and fast ripples $(250-500 \mathrm{~Hz})]$ in low-voltage fast-onset (LVF) and hypersynchronous-onset (HYP) seizures (Velasco et al., 2000; Bragin et al., 2005, 2009; Ogren et al., 2009) during the preictal and ictal periods.

\section{Materials and Methods}

Animal preparation. Male Sprague Dawley rats (250-300 g) were obtained from Charles River and let habituate for $72 \mathrm{~h}$ after delivery before pilocarpine treatment. They were housed under controlled conditions, at $22^{\circ} \mathrm{C}\left( \pm 2^{\circ} \mathrm{C}\right)$ and $12 \mathrm{~h}$ light/dark cycle (lights on from 7:00 A.M. to 7:00 P.M.) with food and water ad libitum. All procedures were approved by the Canadian Council of Animal Care, and all efforts were made to minimize suffering and the number of animals used.

Rats were given injections of scopolamine methylnitrate ( $1 \mathrm{mg} / \mathrm{kg}$, i.p.; Sigma-Aldrich) and, 30 min later, a single dose of pilocarpine hydrochloride (380 mg/kg, i.p.; Sigma-Aldrich) (Bortel et al., 2010; Lévesque et al., 
A

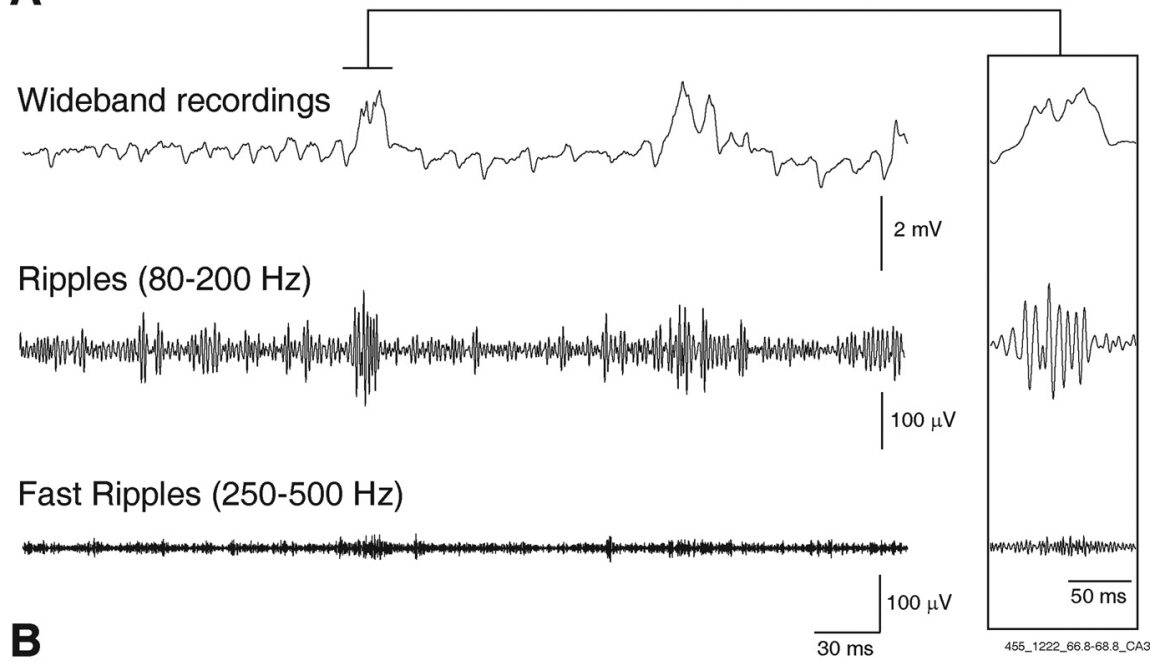

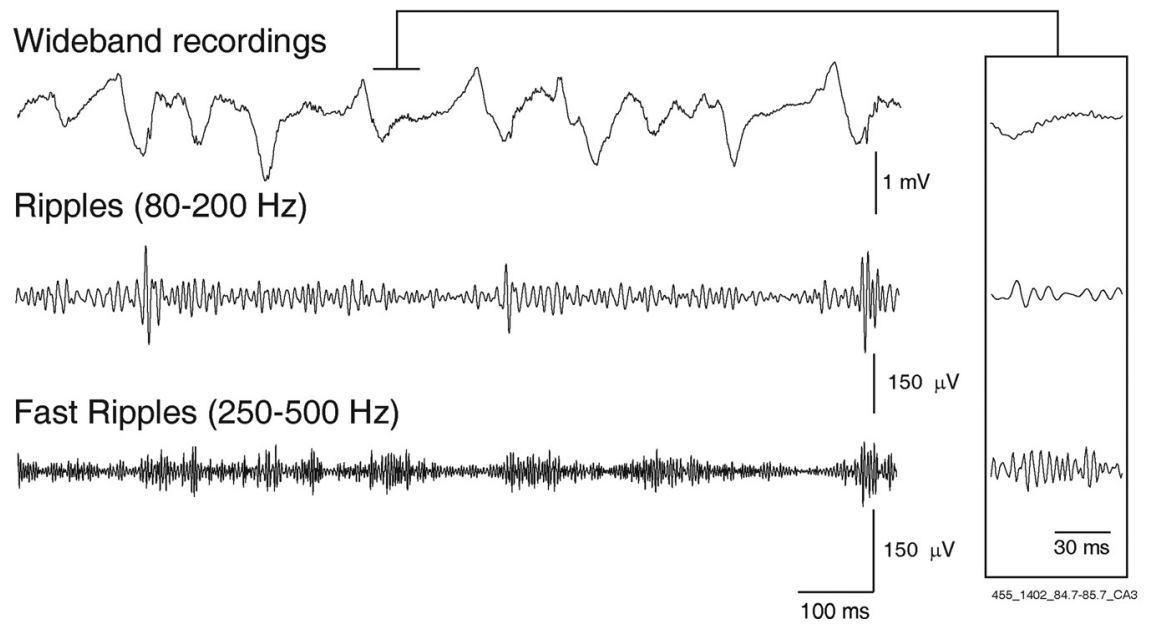

Figure 1. A, Representative local field potentials recorded from the CA3 region during an HYP seizure, 6.8 s after seizure onset showing the occurrence of a ripple $(80-200 \mathrm{~Hz}$ ) (inset). $\boldsymbol{B}$, Representative local field potentials from the $C$ A3 region during another HYP seizure in the same rat, showing the occurrence of a fast ripple $(250-500 \mathrm{~Hz})$ (inset) $24.7 \mathrm{~s}$ after seizure onset.

Local field potential recordings and histological localization of depth electrodes. After surgery, rats were housed individually in custom-made Plexiglas boxes $(30 \times 30 \times 40 \mathrm{~cm})$ and let habituate to the environment for $24 \mathrm{~h}$. The pin connector was then connected to a multichannel cable and electrical swivel (Slip ring T13EEG, Air Precision; or Commutator SL 18C, HRS Scientific) and LFPvideo monitoring ( $24 \mathrm{~h}$ per day) was performed. LFPs were amplified via an interface kit (Mobile 36ch LTM ProAmp; Stellate), low-pass filtered at $500 \mathrm{~Hz}$, and sampled at $2 \mathrm{kHz}$ per channel. Infrared cameras were used to record day/night video files that were time-stamped for integration with the electrophysiological data using monitoring software (Harmonie; Stellate). Throughout the recordings, animals were placed under controlled conditions $\left(22 \pm 2^{\circ} \mathrm{C}, 12 \mathrm{~h}\right.$ light/dark schedule) and provided with food and water ad libitum. LFP-video recordings were performed up to $15 \mathrm{~d}$ after status epilepticus.

At the end of the recording, rats were deeply anesthetized with isoflurane, and electrode localization was aided by lesioning the surrounding tissue by passing current $(500 \mu \mathrm{A}, 120 \mathrm{~s})$ through each recording electrode. Rats were maintained under deep anesthesia with isoflurane and decapitated. Brains were extracted and postfixed with formaldehyde (BioLynx) for at least $48 \mathrm{~h}$ and later placed in a $30 \%$ sucrose-formalin solution for $48 \mathrm{~h}$. They were then frozen in pulverized dry ice and sliced (30 $\mu \mathrm{m}$ thick) using a cryostat. Brain sections were mounted on gelatinized glass slides and stained using a cresyl violet solution. Location of the lesions was evaluated according to the atlas of Paxinos and Watson (1998) [see Lévesque et al. (2011) for the histological localization of electrodes].

Detection of high-frequency oscillatory events. A multiparametric algorithm was used to identify oscillations in each frequency range, using routines based on standardized functions (Matlab Signal Processing Toolbox). For each seizure, raw LFP recordings were bandpass filtered in the $80-200 \mathrm{~Hz}$ and in the $250-500 \mathrm{~Hz}$

2011). Their behavior was scored according to the Racine scale (Racine, 1972), and status epilepticus was defined as continuous stage 5 seizures. Status epilepticus was terminated after $1 \mathrm{~h}$ by injection of diazepam ( 5 $\mathrm{mg} / \mathrm{kg}$, i.p.; CDMV) and ketamine (50 mg/kg, i.p.; CDMV) (Martin and Kapur, 2008). The mortality rate was $13 \%$. Surviving animals were allowed to recover for $72 \mathrm{~h}$ before surgery. They were then anesthetized with isoflurane (3\%) in $100 \% \mathrm{O}_{2}$ and positioned in a stereotaxic frame so that lambda and bregma laid in the same horizontal plane. An incision was made in the skin to expose the skull plate. Four stainless steel screws (2.4 mm length) were fixed to the skull, and four small holes were drilled to allow the implantation of bipolar electrodes $(20-30 \mathrm{k} \Omega ; 30-50 \mathrm{~mm}$ length; distance between exposed tips, $500 \mu \mathrm{m}$ ) made by twisting and gluing two 0.006 inch resin-insulated copper wires. Contacts consisted of the cut edge of the wire $\left(0.018 \mathrm{~mm}^{2}\right.$ ) (Châtillon et al., 2011). Electrodes were implanted in the CA3 subfield of the ventral hippocampus (AP, -4.4 ; ML, $\pm 4 ; \mathrm{DV},-8.8$ ), medial entorhinal cortex (AP, -6.6; ML, \pm 4 ; $\mathrm{DV},-8.8)$, ventral subiculum ( $\mathrm{AP},-6.8 ; \mathrm{ML}, \pm 4 ; \mathrm{DV},-6)$ and dentate gyrus (AP, -4.4 ; ML, \pm 2.4 ; DV, 3.4). Screws and electrode pins were connected with a pin connector and fastened to the skull with dental cement. A cortical screw placed in the frontal bone was used as reference, and a second screw, placed on the opposite side of the frontal region, was used as ground. After surgery, rats received topical chloramphenicol (Erfa) and lidocaine (5\%; Odan) and were given subcutaneous injections of ketoprofen ( $5 \mathrm{mg} / \mathrm{kg}$; Merail), buprenorphine $(0.01-0.05 \mathrm{mg} / \mathrm{kg}$ repeated every $12 \mathrm{~h} ; \mathrm{CDMV}$ ) and $2 \mathrm{ml}$ of $0.9 \%$ sterile saline. frequency range using a finite impulse response filter; zero-phase digital filtering was used to avoid phase distortion. A $10 \mathrm{~s}$ artifact-free period (50-40 s before the seizure onset) was selected as a reference for signal normalization. LFPs from each region were normalized using their own reference period. To be considered as an HFO candidate, oscillatory events in each frequency band had to show at least four consecutive cycles having amplitude of 3 SD above the mean of the reference period. The time lag between two consecutive cycles had to be between 5 and $12.5 \mathrm{~ms}$ for ripples and between 2 and $4 \mathrm{~ms}$ for fast ripples. Furthermore, special care was taken to avoid the detection of false HFOs, since ripples were kept for analysis only if they were visible in the $80-200 \mathrm{~Hz}$ range, whereas fast ripples were kept only if they were visible in the $250-500 \mathrm{~Hz}$ range. Overlapping events, which may be caused by the filtering of sharp spikes (Bénar et al., 2010), were thus excluded from the analysis. Visual validation was also performed to eliminate the false positive created by movement artifacts. If a time period containing movement artifacts was observed on one channel, the same time period was removed on all channels. The final result was a list of "ripples" and "fast ripples," throughout the recording, for each region. Figure 1 shows examples of HFO events, whereas Figures $2 A b, B b, C b$, and $D b$ and $3 A b, B b, C b$, and $D b$ show HFOs during LVF and HYP seizures, respectively. Note that HFOs were visible on the wideband recordings and that there were no overlapping oscillatory events occurring simultaneously in the ripple and fast ripple frequency bands. 
Detection of seizures and seizure-onset zones. Seizures were identified automatically with the ICTA-D seizure detector (Harmonie; Stellate). Validation of the results provided by the detector was performed by visual inspection of LFPs and video. Termination of convulsive seizures was usually followed by wet-dog shakes. LFP recordings showed that seizures were characterized by paroxysmal discharges of increasing amplitude that involved multiple channels, for a duration of at least $5 \mathrm{~s}$.

On average, the first seizure was observed 5.5 $( \pm 2.3)$ days after the pilocarpine induced status epilepticus. We considered the occurrence of the first seizure as the end of the latent period. All seizures used for analysis were thus extracted during the chronic period, up to $15 \mathrm{~d}$ after status epilepticus. Only seizures with goodquality recordings on every channel were selected for analysis. Time periods containing the preictal and the ictal periods were exported for off-line analysis in Matlab 7.9.0 (Mathworks). To identify seizure-onset zones, the power spectral distribution of the LFPs from every region was computed, using a frequency range from 1 to $50 \mathrm{~Hz}$. To enhance the detection of seizure onsets, a gamma correction with a factor 0.15 was applied to the spectrogram to improve the contrast to random noise.

Three reviewers blinded to electrode location were instructed to identify independently one or more seizure-onset zones, by looking at power spectral densities and LFPs. In addition, they were asked to point at the time of seizure onset in each brain structure. When seizure onset was preceded by focal interictal spikes (also termed preictal spikes; see Huberfeld et al., 2011), the onset time was identified as the time of fast-activity onset (Figs. 2, 3, arrows). Whenever reviewers did not agree on the location of the seizure-onset zone or the time of onset, the recordings were further analyzed jointly until agreement was reached. Seizures could be categorized into four types: (1) seizures initiating in CA3 were labeled as "CA3"; (2) those originating from $\mathrm{CA} 3$ and another region simultaneously were termed "CA3+"; (3) seizures initiating simultaneously in all regions were termed "widespread"; and (4) those that did not involve CA3 were labeled as "CA3 -".

Seizures were then transformed into a time scale from 1 (start of seizure) to 100 (end of seizure) to account for differences in seizure duration (range, $18-135 \mathrm{~s}$ ). The preictal period was also transformed into a time scale from 1 to 100 . Values for the ripples and fast ripples were then averaged according to seizure type and region of onset. Distribution histograms of HFO occurrence were built for the preictal and ictal periods.

Classification of seizures. Seizures were classified in two groups according to their onset pattern (Velasco et al., 2000). LVFs were characterized by the occurrence of a positive- or negative-going spike that was followed by the appearance of low-amplitude, high-frequency activity (Fig. 2). HYPs were characterized at onset by a pattern of focal (preictal) spiking at a frequency of $\sim 2 \mathrm{~Hz}$ (Fig. 3).

Statistical analysis. Rates and duration of each type of HFO (ripples, fast ripples) were computed for each region (CA3, entorhinal cortex,

\section{A $\mathrm{CA} 3$}

D Subiculum

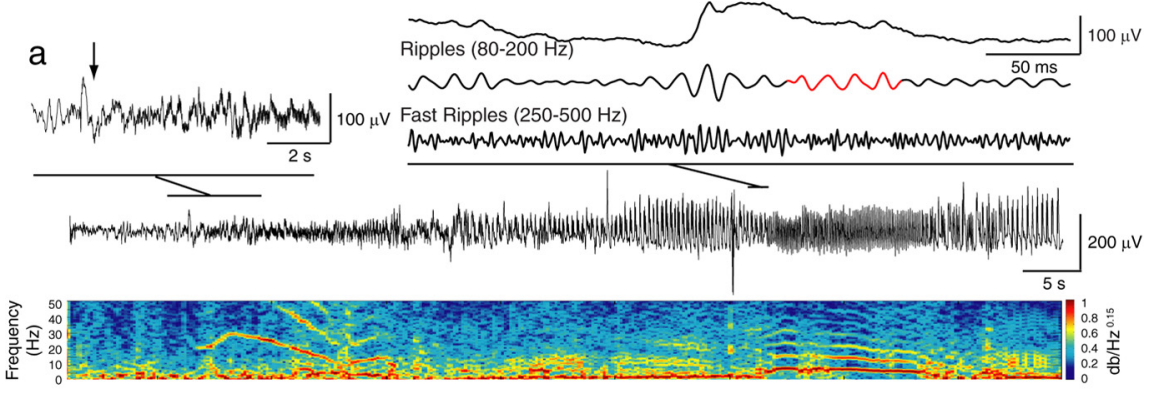

\section{B Entorhinal Cortex}

\section{$\mathrm{b}$ Wideband recordings}

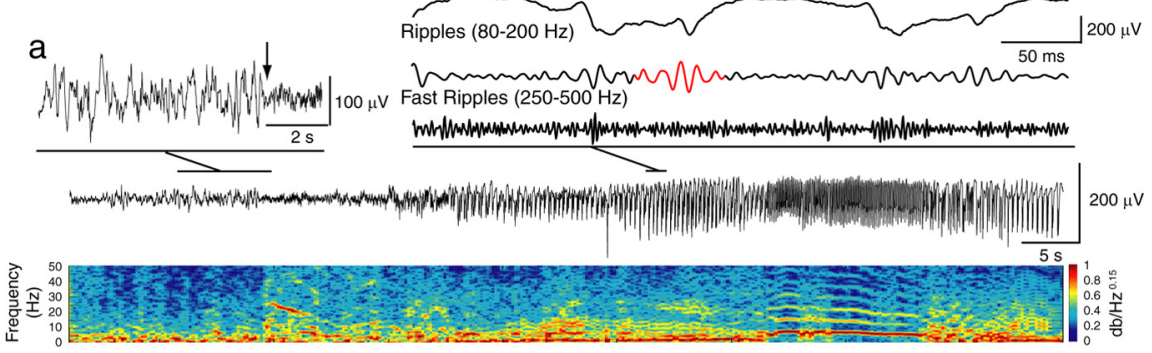

\section{Dentate Gyrus}

b Wideband recordings
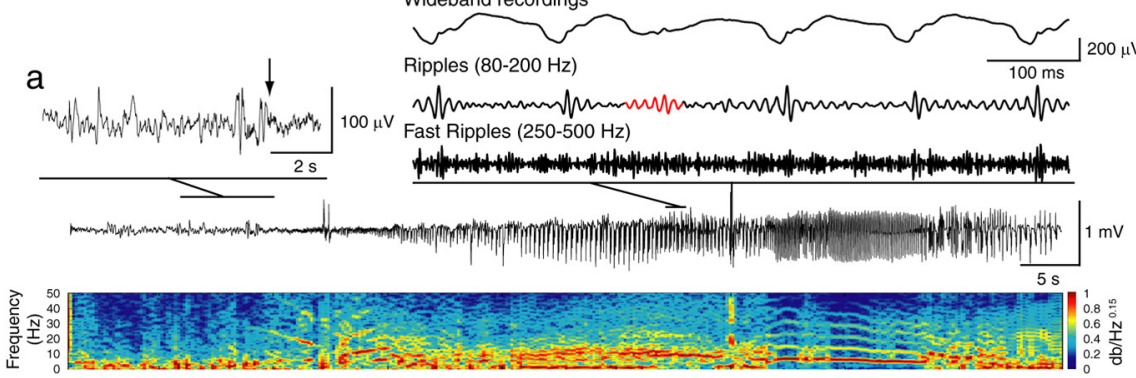

\section{b}
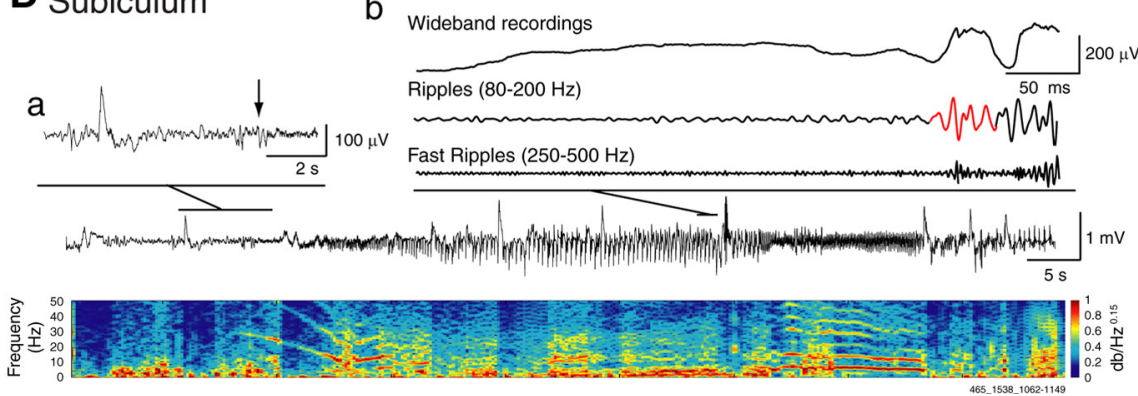

Figure 2. Representative LVF seizure recorded simultaneously from the CA3 region $(\boldsymbol{A})$, the entorhinal cortex $(\boldsymbol{B})$, the dentate gyrus $(\boldsymbol{C})$, and the subiculum $(\boldsymbol{D})$ with corresponding spectrograms. Note in the spectrogram of $\mathrm{CA} 3$ that highly rhythmic activity in the $10-20 \mathrm{~Hz}$ frequency range characterizes the onset of ictal activity; this change was used by the reviewers to identify the seizure-onset time and zone. Note also that the initial phase of the seizure is shown in $\boldsymbol{a}$ on an expanded time scale; arrows indicate the onset of fast activity in CA3 and in the areas of propagation. Wideband and filtered signals in the ripple $(80-200 \mathrm{~Hz})$ and fast ripple $(250-500 \mathrm{~Hz})$ frequency ranges are also shown, with detected HFO highlighted in red (b). Note that HFOs are visible on the wideband unfiltered signal and that they do not always occur on the spike component. dentate gyrus, and subiculum) using the values that were obtained from all seizures. The preictal and ictal periods were divided in three equal parts, and rates of ripples and fast ripples in each part were compared using nonparametric Wilcoxon signed-rank tests followed by Bonferroni-Holm corrections for multiple comparisons. This allowed us to evaluate whether ripples or fast ripples predominated during each seizure type and at specific moments of the preictal and ictal periods, in seizure-onset zones and in regions of secondary 

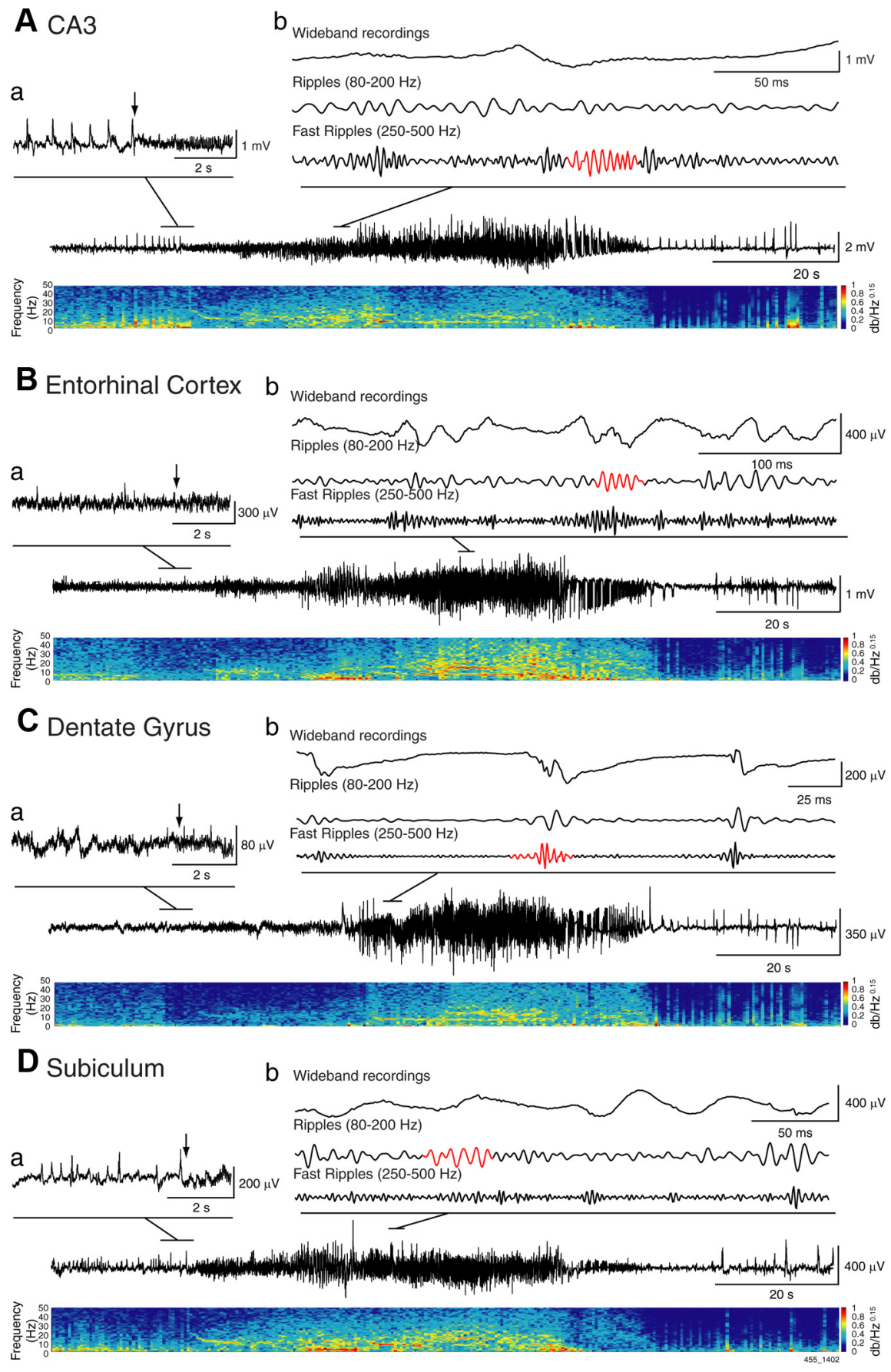

Figure 3. Representative HYP seizure recorded simultaneously from the CA3 region $(\boldsymbol{A})$, the entorhinal cortex $(\boldsymbol{B})$, the dentate gyrus $(\boldsymbol{C}$, and the subiculum $(\boldsymbol{D})$. The initial phase of the seizure on an expanded time scale is shown in $\boldsymbol{a}$; arrows indicate the onset of fast activity in $\mathrm{CA} 3$ and in the areas of propagation. Note that preictal spikes typical of HYP seizures occurred, in most cases, in CA3 and subiculum. Wideband and filtered signals in the ripple $(80-200 \mathrm{~Hz})$ and fast ripple $(250-500 \mathrm{~Hz})$ frequency range are shown in $\boldsymbol{b}$, with HFOs highlighted in red.

spread. Nonparametric Kruskall-Wallis tests were used to compare rates and duration of ripples and fast ripples between seizure types (i.e., LVF and HYP), regions (seizure-onset zones and regions of secondary spread), and time periods (preictal and ictal periods). We also evaluated whether ripple and fast ripple rates increased significantly during the preictal period by applying a linear regression between time and HFO rates. Statistical tests were performed in Matlab 7.9.0 (Mathworks) using the Statistics Toolbox. Results are expressed as mean \pm SEM. The level of significance was set to $p<0.05$.

\section{Results}

\section{Seizure-onset zones}

We analyzed a total of 42 seizures that were recorded in 6 epileptic animals. In one rat, all seizures were CA3; in two rats, they were CA3 in $53.9 \%$ and in $50 \%$ of cases, whereas the rest were $\mathrm{CA} 3+$. In the fourth rat, seizures were distributed as follows: CA3, 18.15\%; CA3+, 36.4\%; widespread, $27.3 \%$; CA3-, 18.15\%. In the fifth rat, $50 \%$ of seizures were widespread and $50 \%$ were CA3. In the sixth rat, seizures were always widespread.

\section{Types of seizure onset}

Representative examples of LVF and HYP seizures are shown in Figures 2 and 3, respectively. Fifty percent ( 21 of 42 ) of seizures were HYP, whereas $42.9 \%$ (18 of 42 ) were LVF. The remaining three seizures $(7.1 \%)$ could not be classified as either HYP or LVF seizures and were excluded from the analysis. Three rats showed both types of seizures, one showed only LVF seizures, and two showed only HYP seizures. The duration of LVF seizures [87.4 $( \pm 7.3) \mathrm{s}$ ] was significantly longer than the duration of HYP seizures [58.1 ( \pm 5.2$) \mathrm{s}$ ] $(p<0.05)$ (Fig. 4A).

Relationship between seizure-onset zone and seizure-onset type

LVF seizures were characterized by a steady buildup of epileptic activity that started in the hippocampus (CA3) in 9 of 18 cases $(50 \%)$, whereas the remaining seizures were widespread (22.2\%), CA3+ (22.2\%), or CA3 - (5.6\%) (Fig. $4 B$ ). On the other hand, $66.6 \%$ of HYP seizures ( 14 of 21) involved CA3 as the seizure-onset zone $(\mathrm{CA} 3$ or $\mathrm{CA} 3+)$ (Fig. $4 \mathrm{~B})$. In six of seven CA3 + HYP seizures, the onset zones were in CA3 and the subiculum; in the remaining case, the onset zone was in CA3 and entorhinal cortex. The remaining seven HYP seizures were labeled as widespread. It should be noted that preictal spiking, characteristic of HYP seizures, only occurred in CA3 or in CA3 and another region simultaneously $(\mathrm{CA} 3+)$ but never outside the hippocampus alone (e.g., the seizure shown in Fig. 3).

Duration of HFOs and relationship with the type of seizure onset

We recorded a total of 19,286 ripples and 19,868 fast ripples. Ripples lasted, on average, $47.9( \pm 0.2) \mathrm{ms}$, whereas fast ripples lasted, on average, $14.8( \pm 0.1) \mathrm{ms}$. When comparing the duration of ripples during the preictal and ictal periods of LVF and HYP seizures, we observed a significant effect of seizure type (LVF and HYP), region (seizure-onset zones and regions of secondary spread), and time periods (preictal and ictal) $\left(\chi^{2}=\right.$ 1002.2; df $=7 ; p<0.001)$. Post hoc comparisons revealed that 
during LVF seizures, (1) ripples lasted longer during the ictal period compared with the preictal period, both in seizure-onset zones and in regions of secondary spread; (2) ripples lasted longer in seizure-onset zones than in regions of secondary spread $(p<0.001)$ (Fig. $5 A$, Ictal period), whereas (3) no significant differences were observed during the preictal period (Fig. 5, Pre-ictal period); and (4) ripples lasted longer during the ictal period of LVF seizures in both seizure-onset zones and regions of secondary spread, compared with those recorded during HYP seizures $(p<0.001)$ (Fig. $5 A$,

Ictal period). The duration of fast ripples did not differ between seizure types, regions, or time periods (Fig. $5 B$ ).

\section{Spatial and temporal distribution patterns of HFOs in temporal lobe regions}

Figure 6 shows the temporal and spatial distribution of HFOs during LVF seizures in each region. Significantly higher ripple rates compared with fast ripple rates were observed in the preictal period in CA3, which was, in most cases (72.2\%), the LVF seizure-onset zone (Fig. 6, CA3, Pre-ictal) $(p<0.001)$. During the ictal period, all regions showed significantly higher ripple rates compared with fast ripple rates $(p<0.001)$ (Fig. 6, Ictal).

Comparison of HFO rates during the preictal period of HYP seizures revealed significantly higher rates of fast ripples compared with ripples $(p<0.001)$ in all regions (Fig. 7, Pre-ictal). During the ictal period, higher fast ripple rates were still observed in all regions compared with ripple rates $(p<0.001)$ (Fig. 7 , Ictal).

\section{Spatial and temporal distribution patterns of HFOs in seizure-onset zones and regions of secondary spread} Comparing ripple and fast ripple rates that occurred during LVF seizures in onset zones and in regions of secondary spread revealed that during the preictal period, the formers increased significantly over time $\left(r^{2}=0.37 ; p<0.0005\right)$ and were higher than fast ripple rates $(p<0.001)$ in seizure-onset zones only (Fig. $8 \mathrm{~A}$, Pre-ictal period). In regions of secondary spread, no significant difference between ripple and fast ripple rates were observed, and HFO rates did not increase significantly over time (Fig. 8 A, Preictal period). During the ictal period, ripple rates continued to predominate over fast ripples in the seizure-onset zone $(p<$ 0.001) (Fig. 8A, Ictal period) and became significantly higher compared with fast ripple rates during the last part of the seizure in regions of secondary spread $(p<0.001)$ (Fig. 8 A, Ictal period). In addition, ripple rates were overall significantly higher in seizure-onset zones than in regions of secondary spread $\left(\chi^{2}=\right.$ 144.33 ; $\mathrm{df}=3 ; p<0.01$ ).

The comparison of HFO rates during HYP seizures revealed that during the preictal period, fast ripple rates had significant and linear increases in both seizure-onset zones $\left(r^{2}=0.37 ; p<\right.$ $0.0005)$ and regions of secondary spread $\left(r^{2}=0.62 ; p<0.0001\right)$ (Fig. $8 B$, Pre-ictal period). Fast ripple rates were also higher than ripple rates during the preictal period $(p<0.001, p<0.05)$ in seizure-onset zones and in regions of secondary spread (Fig. $8 B$, Pre-ictal period). Throughout the ictal period, fast ripple rates were higher than ripple rates in seizure-onset zones and in regions of secondary spread ( $p<0.001)$ (Fig. 8 B, Ictal Period). No significant differences were observed when comparing rates of
B
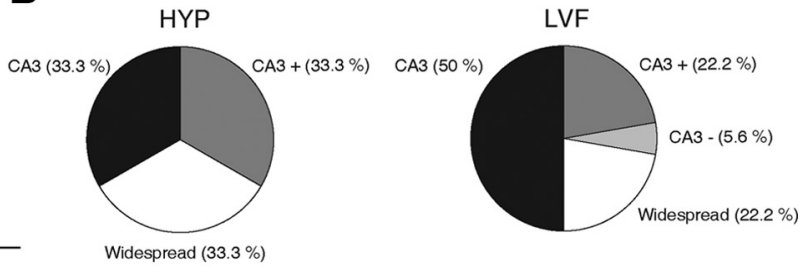

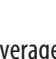

Weiz

Figure 4. $\quad \boldsymbol{A}$, Bar graph showing the average duration of LVF and HYP seizures. HYP seizures were significantly shorter compared with LVF seizures $\left({ }^{*} p<0.05\right), B$, Pie charts showing the percentage of seizures labeled as CA3, CA3 +, CA3 - , and widespread for LVF and HYP seizures. Note that, in most cases, the CA3 was involved as a seizure-onset zone $(66.6 \%$ of HYP seizures and $72.2 \%$ of $\begin{array}{ll}\text { A } & \square \text { Seizure onset zones } \\ \text { Ripples }(80-200 \mathrm{~Hz}) & \\ \text { Pre-ictal period of secondary spread }\end{array}$

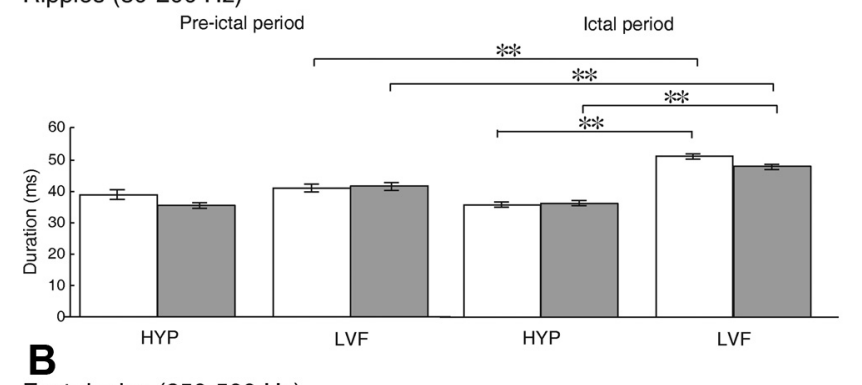

Fast ripples $(250-500 \mathrm{~Hz})$

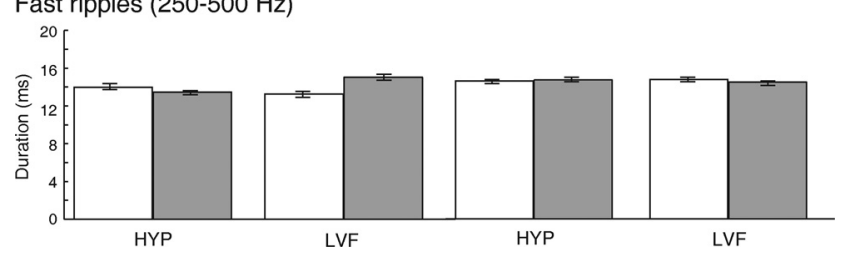

Figure 5. Bargraph showing the duration of ripples $(\boldsymbol{A})$ and fast ripples $(\boldsymbol{B})$ during the preictal and ictal periods for HYP and LVF seizures, in seizure-onset zone and in regions of secondary spread. Note that the duration of ripples was significantly longer during LVF seizures in seizure-onset zones and in regions of secondary spread, compared with HYP seizures. Ripples also lasted longer in seizure-onset zones of LVF seizures compared with regions of secondary spread. During LVF seizures, the duration of ripples was also longer during the ictal period compared with the preictal period. ${ }^{* *} p<0.001$.

fast ripples in seizure-onset zones and in regions of secondary spread throughout the entire seizure.

\section{Discussion}

The main findings of our study can be summarized as follows: (1) spontaneous seizures observed after a pilocarpine-induced status epilepticus are characterized by either LVF or HYP onsets, the former seizures being longer in duration; (2) specific HFO patterns are associated with these two types of seizures since ripples predominate during LVF seizures and fast ripples during HYP seizures; (3) ripple rates in LVF seizures increase over time before onset and are higher than fast ripple rates during both preictal and ictal periods; (4) ripples occurring during LVF seizures have longer duration and higher rates in seizure-onset zones than in regions of secondary spread; (5) ripple duration is longer in seizure-onset zones during the ictal period of LVF seizures compared with the preictal period and the duration of ripples occurring during HYP seizures; (6) HYP seizures are characterized by an increase over time of fast ripple rates before seizure onset that remains higher than ripple rates throughout the ictal period. 
Low-voltage fast-onset seizures
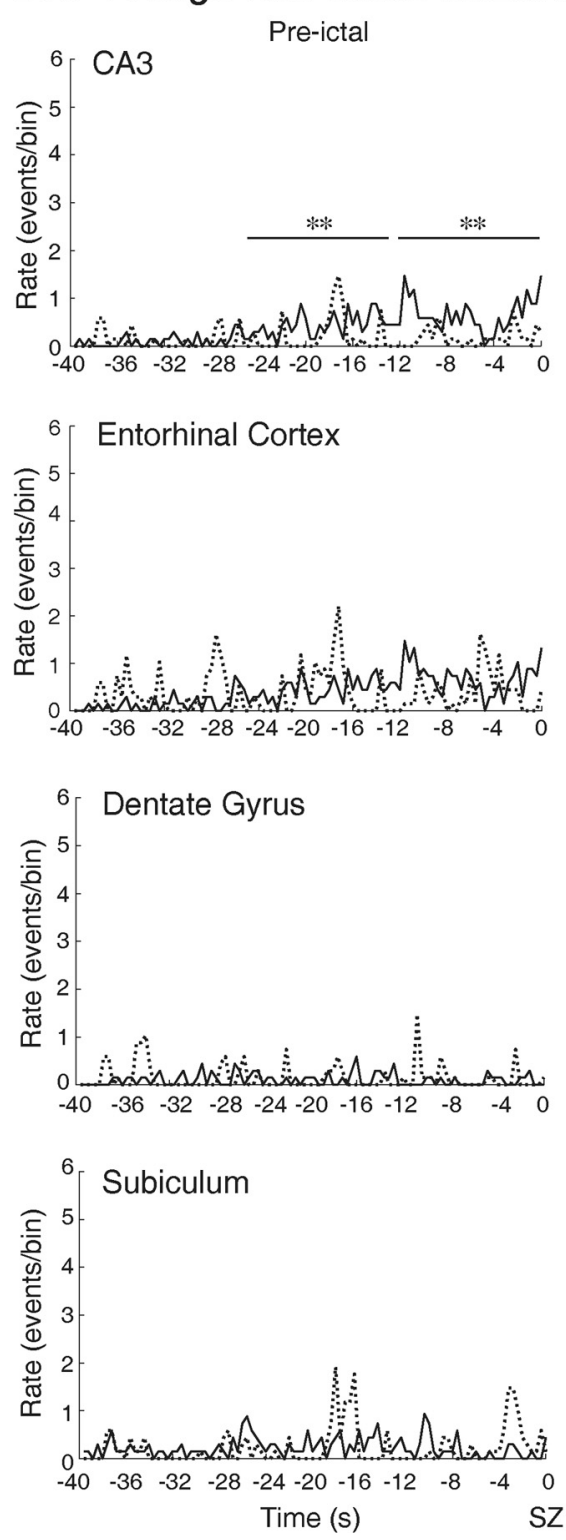

Figure 6. Spatial and temporal distribution patterns of HFOs during LVF seizures in CA3, entorhinal cortex, dentate gyrus, and subiculum. Ripple rates were higher compared with fast ripples rates during the preictal period of LVF seizures, in CA3, which was, in most cases, the seizure-onset zone. During the ictal period, all regions showed high rates of ripple compared with fast ripple rates. ${ }^{* *} p<0.001$.

\section{Seizure-onset patterns as a determinant of seizure duration}

Spontaneous seizures occurring in pilocarpine-treated epileptic rats are characterized by either LVF or HYP onsets. These two types of seizure onset were originally identified in temporal lobe patients recorded with intracranial depth electrodes (Velasco et al., 2000) and later confirmed in rats made epileptic by intrahippocampal kainic acid injection (Bragin et al., 2005). The probability of occurrence of LVF and HYP seizures was indeed similar in our experiments, and both seizure types could be recorded in three rats. However, we discovered that LVF seizures lasted significantly longer than HYP seizures. The cellular and pharmacological mechanisms responsible for such difference remain to be established, but retrospective analysis of in vitro data obtained in the entorhinal cortex during application of the $\mathrm{K}^{+}$-channel blocker 4 -aminopyridine suggests a role for $\mathrm{GABA}_{\mathrm{A}}$ receptor signaling be- cause (1) ictal events characterized by an LVF-like onset pattern (Lopantsev and Avoli, 1998a) last longer than those preceded by continuous interictal spiking (Lopantsev and Avoli, 1998b) and (2) blocking ionotropic glutamatergic transmission in these experiments reveals the presence of synchronous GABA receptor-mediated potentials only in brain slices that generate LVF-like onset discharges (Lopantsev and Avoli, 1998a,b).

\section{Fast ripples and ripples characterize different seizure-onset patterns}

We have also found in this model of temporal lobe epilepsy that the pattern of $\mathrm{HFO}$ occurrence is related to seizureonset type. It has been reported in epileptic patients that the occurrence of HYP seizures may be related to hippocampal atrophy and gliosis since patients presenting with LVF seizures show more diffuse neuronal loss (Park et al., 1996; Velasco et al., 2000; Staba et al., 2007; Ogren et al., 2009). However, our results do not favor the hypothesis that neuronal loss defines the type of seizure onset (i.e., HYP and LVF) because three of six rats showed both types of seizures. Hence, our findings indicate that HFOs reflect dynamic processes that may rest on the functional organization of neuronal clusters during the ictal period rather than the level of neuronal damage, which in our experimental model was induced by the initial pilocarpine-induced status epilepticus.

The occurrence of distinct types of HFOs during HYP and LVF seizures could also depend on the location of the seizure-onset zone. Previous studies have shown that HYP seizures are rather restricted in origin and often start in the hippocampus, whereas LVF seizures initiate from widespread regions often involving extrahippocampal areas (Velasco et al., 2000; Bragin et al., 2005). This evidence would explain the high rates of fast ripples recorded during HYP seizures and their relative absence during LVF seizures, assuming that fast ripples occur in areas that are located in or near the epileptic generator (Bragin et al., 1999; Staba et al., 2002; Jirsch et al., 2006, 2010b). However, we have observed that ripples could also act as markers of seizure-onset zones when the type of seizure onset is considered since ripples increased over time before the initiation of LVF seizures; in addition, ripples occurred at higher rates compared with fast ripple rates both during the preictal and ictal periods in LVF seizure-onset zones. In contrast, fast ripples mainly characterized HYP seizures. Therefore, in temporal lobe regions, before and during ictal events, both ripples and fast ripples would be pathological events, as they would reflect the activity of distinct neural networks that eventually give rise to the different 
seizure-onset patterns observed on the EEG in the clinical context.

\section{Fast ripples and ripples reflect neural} network dynamics during seizures

Our results support the hypothesis that HFOs may trigger seizures (Traub et al., 2001), since ripple rates increased during the preictal period of LVF seizures and fast ripple rates increased a few seconds before HYP seizure onset. Although the exact mechanisms underlying the generation of ripples and fast ripples are not well defined, previous work has suggested that the generation of HFOs characterized by different frequencies rests on specific neuronal mechanisms. Ripples at frequencies up to $200 \mathrm{~Hz}$, which can also be recorded in conditions of physiological excitability, have been proposed to represent IPSP populations generated by principal neurons entrained by network of synchronously active interneurons (Buzsáki et al., 1992; Ylinen et al., 1995). In contrast, fast ripples (at $250-500 \mathrm{~Hz}$ ) do not depend on inhibitory transmission since they are recorded during $\mathrm{GABA}_{\mathrm{A}}$ receptor blockade and are believed to reflect the synchronous firing of principal (glutamatergic) neurons (Dzhala and Staley, 2004; Engel et al., 2009; Bragin et al., 2011). In addition, it has been proposed that fast ripples may result from the loss of synchronization during jittery, out-of-phase burst firing of principal cells in the epileptic hippocampus (Foffani et al., 2007; Ibarz et al., 2010). Hence, the preferential association of ripples or fast ripples with LVF and HYP seizures in the pilocarpine model of temporal lobe epilepsy strongly suggest that specific mechanisms underlying network synchronization are linked to each seizure type.

As mentioned above, LVF seizures recorded in our study shared similar morphological features with a type of ictal-like discharge that is induced in vitro by application of 4-aminopyridine. These ictal-like discharges, which initiate with a long-lasting depolarization of principal neurons followed by fast activity, are thought to be related to an inhibitory process with enhancement of interneuronal activity, mediated by GABAergic signaling at seizure onset (Avoli and de Curtis, 2011). Thus, ripples recorded during these in vitro seizure-like discharges could reflect summated IPSPs generated by pyramidal cells in response to inhibitory interneuron firing. In line with this hypothesis, we have recently reported high rates of ripples along with the virtual absence of fast ripples during ictal-like events generated by piriform cortex networks after the administration of 4-aminopyridine (Panuccio et al., 2012). Therefore, evidence obtained in vitro support the hypothesis that LVF seizures rely on interneuronal activation and GABAergic synchronization.
Hypersynchronous-onset seizures

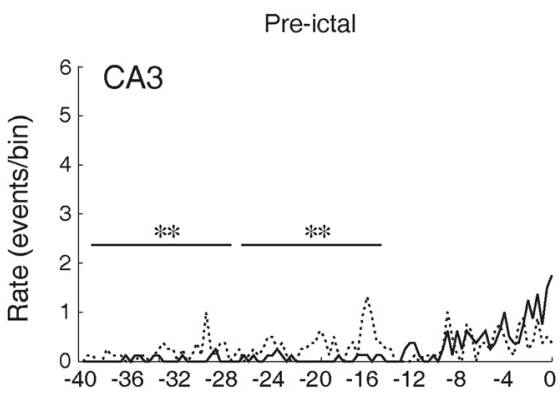

- Ripples $(80-200 \mathrm{~Hz})$

… Fast Ripples $(250-500 \mathrm{~Hz})$
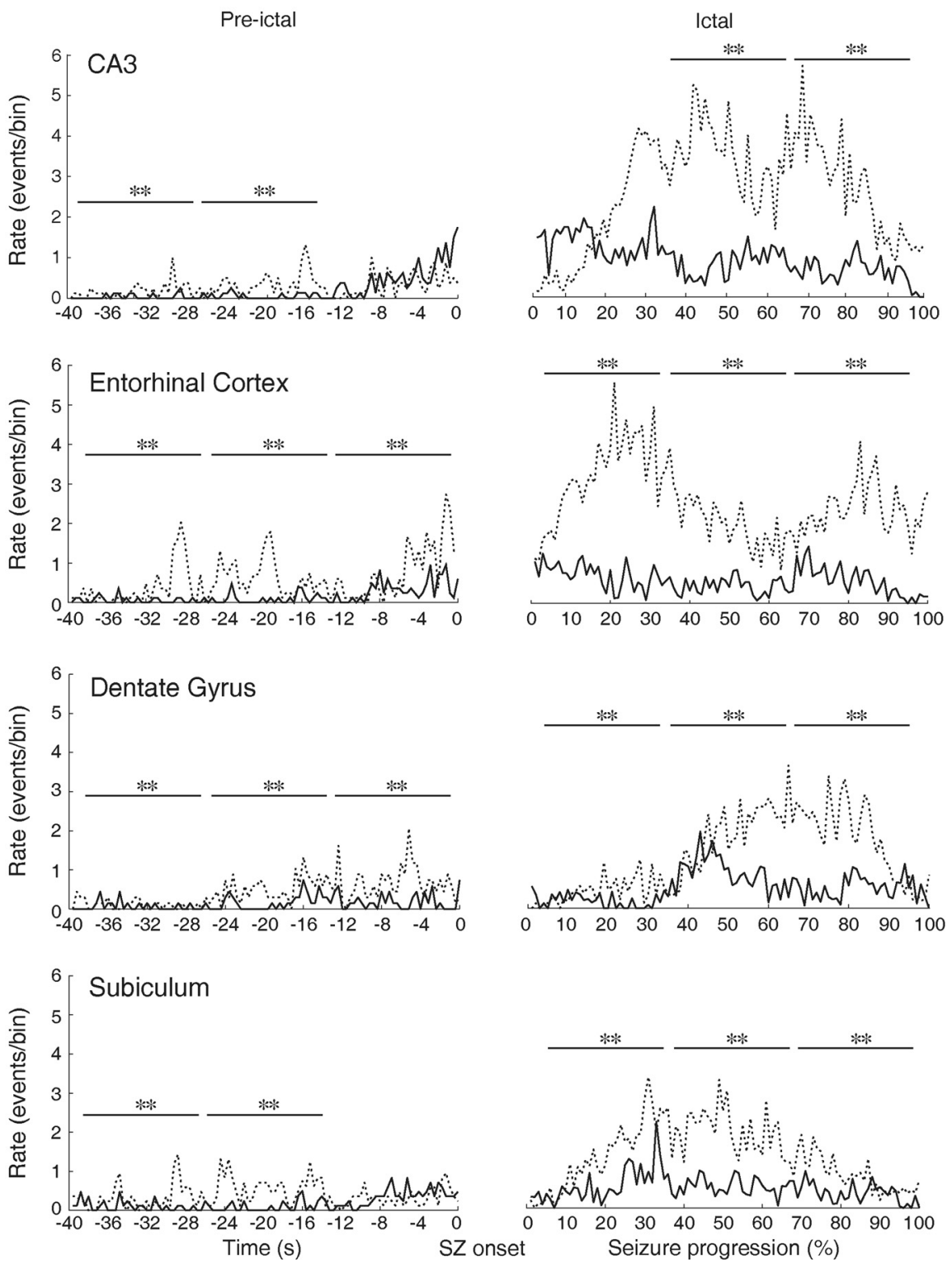

Figure 7. Spatial and temporal distribution patterns of HFOs during an HYP seizure in CA3, entorhinal cortex, dentate gyrus, and subiculum. Fast ripple rates were higher compared with ripple rates in all regions during the preictal and ictal periods. ${ }^{* *} p<0.001$.

\section{Conclusions}

From a clinical perspective, our study emphasizes the need to distinguish seizure-onset patterns in the analysis of HFOs during the preictal and ictal periods. Our findings also put further emphasis on the existence of different types of seizure onset that can co-occur in the same animal over time and that presumably results from different underlying pathophysiological mechanisms of seizure onset. Additional studies should investigate whether fast ripples and ripples show distinct patterns of occurrence during interictal periods depending on the occurrence over time of different seizure types and whether HFOs recorded during interictal events reflect the same mechanisms as HFOs recorded during ictal events. Studies should also investigate the cellular mechanisms and relate single-cell activity to HFOs and seizure-onset patterns. Combining these information together could help to delineate better therapeutical targets in the treatment of temporal lobe epilepsy. 
A Low-voltage fast-onset seizures
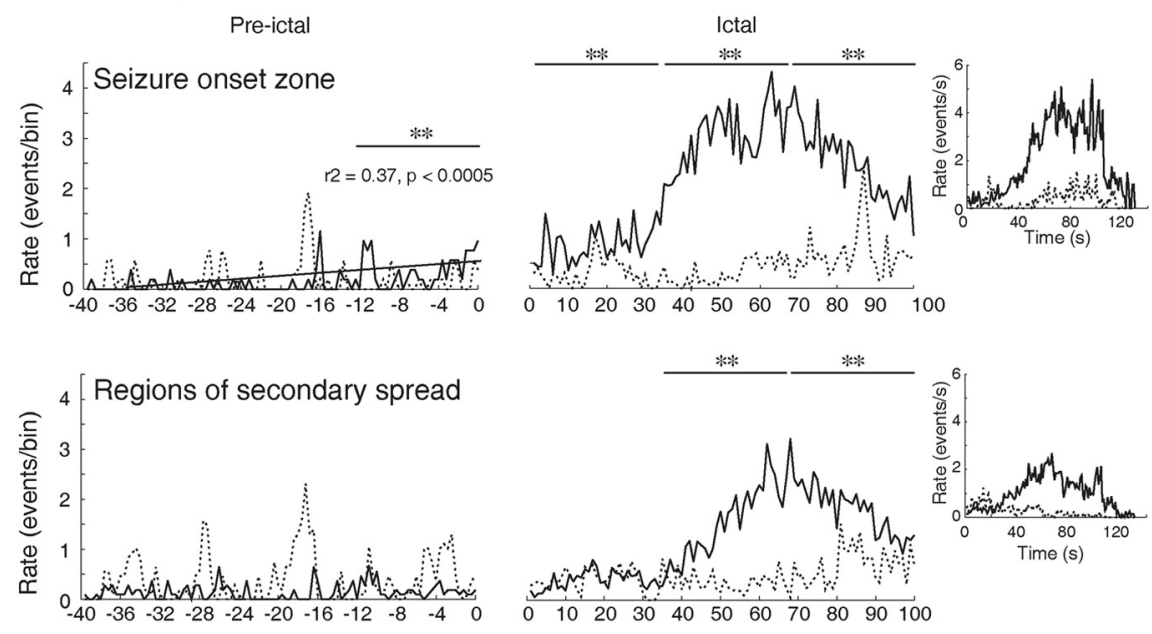

B Hypersynchronous-onset seizures
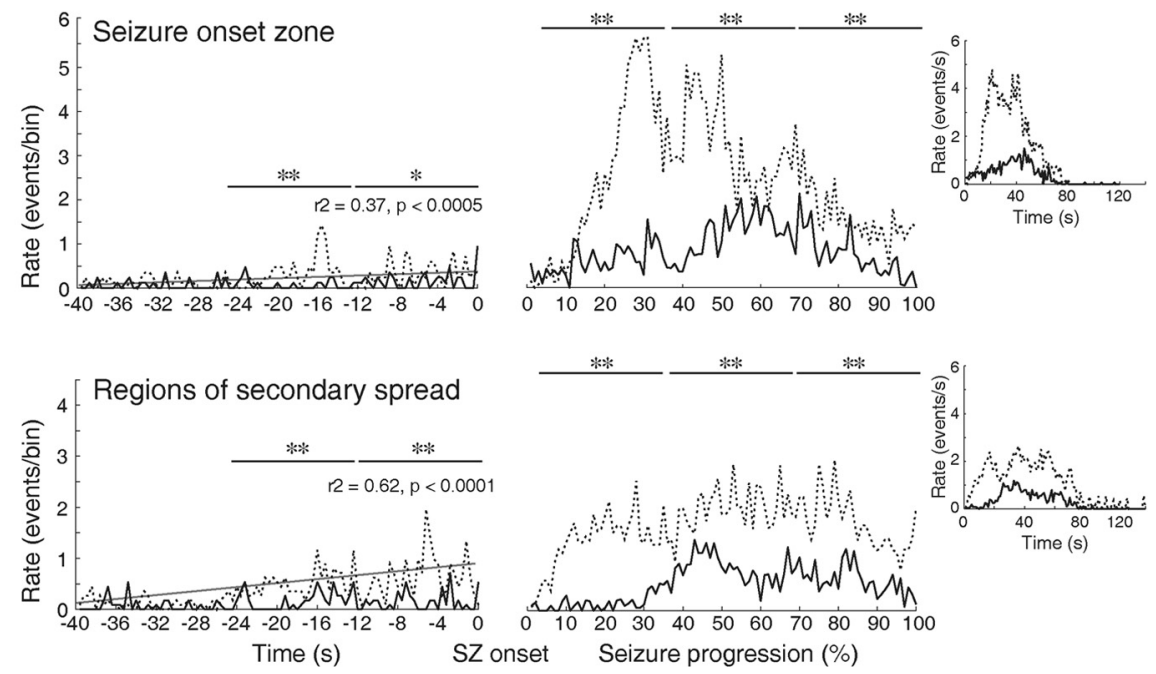

Figure 8. Spatial and temporal distribution patterns of HFOs during $\operatorname{LVF}(\boldsymbol{A})$ and $\mathrm{HYP}(\boldsymbol{B})$ seizures in seizure-onset zones and in regions of secondary spread. $\boldsymbol{A}$, During the preictal period of LVF seizures, in seizure-onset zones, significantly higher rates of ripples were observed compared with fast ripple rates. Moreover, we observed a significant increase of ripple rates over time. During the ictal period, in the seizure-onset zone, the same pattern was observed, as ripple rates were higher compared with fast ripple rates. In regions of secondary spread, ripple rates were higher compared with fast ripple rates, but this occurred later during the seizure. $\boldsymbol{B}$, During the preictal period of HYP seizures, fast ripple rates were higher compared with ripple rates in the seizureonset zone and in regions of secondary spread, and a significant increase of fast ripple rates was observed over time. During the ictal period, the same pattern was observed, as fast ripples were predominant compared with ripples. Insets show rates of HFOs occurring over seconds occurring throughout all seizures recorded. ${ }^{*} p<0.05 ;{ }^{* *} p<0.001$.

\section{References}

Avoli M, de Curtis M (2011) GABAergic synchronization in the limbic system and its role in the generation of epileptiform activity. Prog Neurobiol 95:104-132.

Bénar CG, Chauvière L, Bartolomei F, Wendling F (2010) Pitfalls of high-pass filtering for detecting epileptic oscillations: a technical note on "false" ripples. Clin Neurophysiol 121:301-310.

Bortel A, Lévesque M, Biagini G, Gotman J, Avoli M (2010) Convulsive status epilepticus duration as determinant for epileptogenesis and interictal discharge generation in the rat limbic system. Neurobiol Dis 40:478-489.

Bragin A, Engel J Jr, Wilson CL, Vizentin E, Mathern GW (1999) Electrophysiological analysis of a chronic seizure model after unilateral hippocampal KA injection. Epilepsia 40:1210-1221.

Bragin A, Wilson CL, Almajano J, Mody I, Engel J Jr (2004) High-frequency

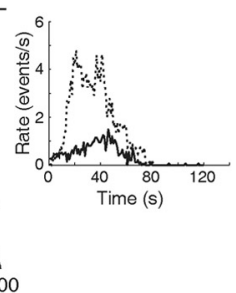

oscillations after status epilepticus: epileptogenesis and seizure genesis. Epilepsia 45: 1017-1023.

Bragin A, Azizyan A, Almajano J, Wilson CL, Engel J Jr (2005) Analysis of chronic seizure onsets after intrahippocampal kainic acid injection in freely moving rats. Epilepsia 46:1592-1598.

Bragin A, Azizyan A, Almajano J, Engel J Jr (2009) The cause of the imbalance in the neuronal network leading to seizure activity can be predicted by the electroencephalographic pattern of the seizure onset. J Neurosci 29:3660-3671.

Bragin A, Benassi SK, Kheiri F, Engel J Jr (2011) Further evidence that pathologic high-frequency oscillations are bursts of population spikes derived from recordings of identified cells in dentate gyrus. Epilepsia 52:45-52.

Buzsáki G, Horváth Z, Urioste R, Hetke J, Wise K (1992) High-frequency network oscillation in the hippocampus. Science 256:1025-1027.

Châtillon CE, Zelmann R, Bortel A, Avoli M, Gotman J (2011) Contact size does not affect high frequency oscillation detection in intracerebral EEG recordings in a rat epilepsy model. Clin Neurophysiol 122:1701-1705.

Crépon B, Navarro V, Hasboun D, Clemenceau S, Martinerie J, Baulac M, Adam C, Le Van Quyen M (2010) Mapping interictal oscillations greater than $200 \mathrm{~Hz}$ recorded with intracranial macroelectrodes in human epilepsy. Brain 133:33-45.

Dzhala VI, Staley KJ (2004) Mechanisms of fast ripples in the hippocampus. J Neurosci 24:8896-8906.

Engel J Jr, da Silva FL (2012) High-frequency oscillations-where are we and where we need to go. Prog Neurobiol. Advance online publication. Retrieved September 4, 2012. http://dx.doi.org/ 10.1016/j.pneurobio.2012.02.001.

Engel J Jr, Bragin A, Staba R, Mody I (2009) High-frequency oscillations: what is normal and what is not? Epilepsia 50:598-604.

Foffani G, Uzcategui YG, Gal B, Menendez de la Prida L (2007) Reduced spike-timing reliability correlates with the emergence of fast ripples in the rat epileptic hippocampus. Neuron 55:930-941.

Huberfeld G, Menendez de la Prida L, Pallud J, Cohen I, Le Van Quyen M, Adam C, Clemenceau S, Baulac M, Miles R (2011) Glutamatergic pre-ictal discharges emerge at the transition to seizure in human epilepsy. Nat Neurosci 14:627-634.

Ibarz JM, Foffani G, Cid E, Inostroza M, Menendez de la Prida L (2010) Emergent dynamics of fast ripples in the epileptic hippocampus. J Neurosci 30:16249-16261.

Jacobs J, Levan P, Chander R, Hall J, Dubeau F, Gotman J (2008) Interictal high-frequency oscillations $(80-500 \mathrm{~Hz})$ are an indicator of seizure onset areas independent of spikes in the human epileptic brain. Epilepsia 49:1893-1907.

Jacobs J, Levan P, Châtillon CE, Olivier A, Dubeau F, Gotman J (2009) High frequency oscillations in intracranial EEGs mark epileptogenicity rather than lesion type. Brain 132:1022-1037.

Jacobs J, Zijlmans M, Zelmann R, Chatillon CE, Hall J, Olivier A, Dubeau F, Gotman J (2010) High-frequency electroencephalographic oscillations correlate with outcome of epilepsy surgery. Ann Neurol 67:209-220.

Jefferys JG, Menendez de la Prida L, Wendling F, Bragin A, Avoli M, Timofeev I, Lopes da Silva FH (2012) Mechanisms of physiological and epileptic HFO generation. Prog Neurobiol. Advance online publication. Retrieved September 4, 2012. http://dx.doi.org/10.1016/j. pneurobio.2012.02.005. 
Jirsch JD, Urrestarazu E, Levan P, Olivier A, Dubeau F, Gotman J (2006) High-frequency oscillations during human focal seizures. Brain 129:1593-1608.

Jiruska P, Csicsvari J, Powell AD, Fox JE, Chang WC, Vreugdenhil M, Li X, Palus M, Bujan AF, Dearden RW, Jefferys JG (2010a) High-frequency network activity, global increase in neuronal activity, and synchrony expansion precede epileptic seizures in vitro. J Neurosci 21:5690-5701.

Jiruska P, Finnerty GT, Powell AD, Lofti N, Cmejla R, Jefferys JG (2010b) Epileptic high-frequency network activity in a model of non-lesional temporal lobe epilepsy. Brain 133:1380-1390.

Khosravani H, Pinnegar CR, Mitchell JR, Bardakjian BL, Federico P, Carlen PL (2005) Increased high-frequency oscillations precede in vitro low-Mg seizures. Epilepsia 46:1188-1197.

Lévesque M, Bortel A, Gotman J, Avoli M (2011) High-frequency oscillations $(80-500 \mathrm{~Hz})$ and epileptogenesis in temporal lobe epilepsy. Neurobiol Dis 42:231-241.

Lopantsev V, Avoli M (1998a) Participation of $\mathrm{GABA}_{\mathrm{A}}$-mediated inhibition in ictal-like discharges in the rat entorhinal cortex. J Neurophysiol 79:352-360.

Lopantsev V, Avoli M (1998b) Laminar organization of epileptiform discharges in the rat entorhinal cortex in vitro. J Physiol 509:785-796.

Martin BS, Kapur J (2008) A combination of ketamine and diazepam synergistically controls refractory status epilepticus induced by cholinergic stimulation. Epilepsia 49:248-255.

Ogren JA, Wilson CL, Bragin A, Lin JJ, Salamon N, Dutton RA, Luders E, Fields TA, Fried I, Toga AW, Thompson PM, Engel J Jr, Staba RJ (2009) Three-dimensional surface maps link local atrophy and fast ripples in human epileptic hippocampus. Ann Neurol 66:783-791.

Panuccio G, Sanchez G, Lévesque M, Salami P, De Curtis M, Avoli M (2012) On the ictogenic properties of the piriform cortex in vitro. Epilepsia 53:459-468.
Park YD, Murro AM, King DW, Gallagher BB, Smith JR, Yaghmai F (1996) The significance of ictal depth EEG patterns in patients with temporal lobe epilepsy. Electroencephalogr Clin Neurophysiol 99:412-415.

Paxinos G, Watson C (1998) The rat brain in stereotaxic coordinates, Ed 4. San Diego: Academic.

Racine RJ (1972) Modification of seizure activity by electrical stimulation. II. Motor seizure. Electroencephalogr Clin Neurophysiol 32:281-294.

Staba RJ, Wilson CL, Bragin A, Fried I, Engel J Jr (2002) Quantitative analysis of high-frequency oscillations $(80-500 \mathrm{~Hz})$ recorded in human epileptic hippocampus and entorhinal cortex. J Neurophysiol 88:1743-1752.

Staba RJ, Frighetto L, Behnke EJ, Mathern GW, Fields T, Bragin A, Ogren J, Fried I, Wilson CL, Engel J Jr (2007) Increased fast ripple to ripple ratios correlate with reduced hippocampal volumes and neuron loss in temporal lobe epilepsy patients. Epilepsia 48:2130-2138.

Traub RD, Whittington MA, Buhl EH, LeBeau FE, Bibbig A, Boyd S, Cross H, Baldeweg T (2001) A possible role for gap junctions in generation of very fast EEG oscillations preceding the onset of, and perhaps initiating, seizures. Epilepsia 42:153-170.

Velasco AL, Wilson CL, Babb TL, Engel J Jr (2000) Functional and anatomic correlates of two frequently observed temporal lobe seizure-onset patterns. Neural Plast 7:49-63.

Wu JY, Sankar R, Lerner JT, Matsumoto JH, Vinters HV, Mathern GW (2010) Removing interictal fast ripples on electrocorticography linked with seizure freedom in children. Neurology 75:1686-1694.

Ylinen A, Bragin A, Nádasdy Z, Jandó G, Szabó I, Sik A, Buzsáki G (1995) Sharp wave-associated high-frequency oscillation $(200 \mathrm{~Hz})$ in the intact hippocampus: network and intracellular mechanisms. J Neurosci 15:30-46.

Zijlmans M, Jacobs J, Kahn YU, Zelmann R, Dubeau F, Gotman J (2011) Ictal and interictal high frequency oscillations in patients with focal epilepsy. Clin Neurophysiol 122:664-671. 